\title{
Revascularização Cirúrgica após Reperfusão no Infarto Agudo do Miocárdio
}

Luiz Alberto MATTOS*, Leopoldo S. PIEGAS*, Ibraim Francisco PINTO*, Luiz Fernando Leite TANAJURA*, Amanda Guerra de MORAES ${ }^{\star}$, Wilson PIMENTEL*, Jorge BUCHLER ${ }^{\star}$, Jarbas J. DINJUYSEN*, Luiz Carlos Bento de SOUZA*, Paulo P. PAULISTA*, Adib D. JATENE**, J. Eduardo M. R. SOUSA*

RBCCV 44205-28

MATTOS, L. A.; PIEGAS, L. S.; PINTO, I. F.; TANAJURA, L. F. L.; MORAES, A. G.; PIMENTEL, W. BUCHLER, J.; DINKHUYSEN, J. J.; SOUZA, L. C. B.; PAULISTA, P. P.; JATENE, A. D.; SOUSA J. E. M. R. - Revascularizaçāo cirúrgica após reperfusão no infarto agudo do miocárdio. Rev. Bras. Cir, Cardiovasc., 20(2): 98-101, 1987.

RESUMO: A cirurgia de revascularização do miocárdio (CRM) é freqüentemente empregada como método complementar no tratamento da lesão coronária residual, após a reperfusão, no infarto agudo do miocárdio (IAM). Pode ser feita tanto na fase aguda como tardiamente. Neste trabalho, são analisados os resultados obtidos em 45/159 (28\%) pacientes (p) submetidos a CRM após reperfusão miocárdica, com sucesso. Foram divididos em dois grupos, segundo o método de reperfusão utilizado: I) 27 (60\%) reperfundidos com estreptoquinase (STK), sendo intracoronária em $22(81 \%)$ e intravenosa em $5(18 \%)$; II) $18(40 \%)$ reperfundidos através da angioplastia coronária (AC), isolada em $10(55 \%)$ e precedida de STK em $8(45 \%)$. Estes pacientes foram analisados quanto ao momento da cirurgia, à evoluão clínica, reestudos angiográficos, avaliação da função ventricular e óbitos. Os mesmos foram seguidos num período de 2 a 55 meses. Os autores discutem os achados encontrados, assim como a capacidade da CRM de complementar a reperfusão, com sucesso, no IAM.

DESCRITORES: infarto do miocárdio, cirurgia; revascularização do miocárdio, cirurgia.

\section{INTRODUÇÃO}

A reperfusão coronária tem sido o método proposto para o tratamento do infarto agudo do miocárdio (IAM), visando diminuir a área de infarto, manter a função ventricular e reduzir a morbimortalidade. A reperfusão deve ser precoce e rápida, na tentativa de preservar a maior quantidade possível de miocárdio viável, obtendo-se, assim, melhores resultados ${ }^{2,3}$.

A desobstrução coronária no IAM pode ser realizada através do emprego de substâncias trombolíticas, como a estreptoquinase, ou pela angioplastia coronária ( $A C)$ isolada, técnica essa de emprego mais recente ${ }^{4}$. No passado, a cirurgia de revascularização do miocárdio (CRM) também foi utilizada como método inicial de reperfusão miocárdica ${ }^{1}$.
Atualmente, a CRM é empregada apenas quando se faz necessário tratar a lesão residual e/ou revascularizar outras áreas isquêmicas não relacionadas ao infarto em evolução ${ }^{6,11,12}$.

O objetivo do presente trabalho é o de analisar os resultados imediatos e tardios obtidos com o emprego da CRM após reperfusāo coronária, com sucesso, no IAM, através do uso prévio da STK e da AC, isoladas, ou associadas.

\section{MATERIAL E MÉTODOS}

Entre julho de 1981 e janeiro de 1987, 208 pacientes internados com IAM submeteram-se a reperfusão coronária, obtendo-se sucesso pela recanalização do vaso

Trabalho realizado no Instituto Dante Pazzanese de Cardiologia. São Paulo, SP, Brasil.

Apresentado ao 14: Congresso Nacional de Cirurgia Cardiaca, Salvador, BA, 27 e 28 de março, 1987.

- Do Instituto Dante Pazzanese de Cardiologia.

*. Do Instituto do Coração da Associaçāo do Sanatório Sírio.

Endereço para separatas: Luiz Alberto Mattos. Caixa Postal 215 - 01000, Såo Pąulo, SP, Brasil 
MATTOS, L. A.; PIEGAS, L. S.; PINTO, I. F.; TANAJURA, L. F. L.; MORAES, A. G.: PIMENTEL, W.: BUCHLER, J.: DINKHUYSEN. J. J.; SOUZA, L, C. B.; PAULISTA, P. P.; JATENE, A. D.; SOUSA, J. E. M. R. - Revascularização cirúrgica após reperfusão no infarto agudo do miocárdio. Rev. Bras. Cir. CardiovasC., 20(2):98-101, 1987.

ocluído, em 159 (76\%). Destes, $45(28 \%)$ realizaram CRM, sendo na fase hospitalar em $36(80 \%)$ e tardiamente, após a alta hospitalar, em $9(20 \%)$. O seguimento destes pacientes variou de 2 a 55 meses (média de 27 meses). Foram avaliados, quanto à sua evolução clínica, reestudos angiográficos e mortalidade hospitalar e tardia.

Foram divididos em dois grupos, conforme o método de reperfusāo utilizado:

Grupo I - $27(60 \%)$ reperfundidos com STK intracoronária em $22(81 \%)$ e intravenosa em 5 (18\%).

Grupo II $-18(40 \%)$ reperfundidos com $A C$, isolada em $10(55 \%)$ e precedida de STK em $8(45 \%)$.

No Grupo I a indicação cirúrgica foi devida à presença de doença multiarterial em 25 (93\%) e por doença uniarterial em 2 (7\%). No Grupo II, a indicaçāo cirúrgica foi por presença de doença multiarterial em 15 (83\%) e unilateral em $3(17 \%)$. No Grupo II, $3(17 \%)$ eram insucessos primários, $6(34 \%)$ desenvolveram reestenose na evolução, em $2(11 \%)$ a recanalização obtida foi insatisfatória e $7(38 \%)$ eram portadores de lesōes multiarteriais.

No Grupo I, o intervalo entre a reperfusão e a CRM variou de 1 a 30 dias (média $=9$ dias), nos 24 pacientes operados na fase hospitalar. Destes, 8 (33\%) o foram nas primeiras 24 horas. A cirurgia foi realizada na fase tardia, após a alta hospitalar, em 3 pacientes, no 2 ., 5 : e 20: meses (média $=9$ meses). No Grupo I realizaram-se 60 pontes de safena ( 15 para descendente anterior, 9 para ramos diagonais, 20 para marginal esquerda e 16 para coronária direita) e 5 anastomoses da artéria mamária interna esquerda para a descendente anterior. O número médio de enxertos por pacientes foi de 2,4.

No Grupo II, o intervalo para realização da CRM variou de 1 a 30 dias (média $=10$ dias), nos 12 pacientes operados na fase hospitalar. Destes, $3(25 \%)$ o foram nas primeiras 24 horas. A cirurgia foi realizada na fase tardia, após a alta hospitalar, em 6 pacientes, no 4 ., $5:, 7^{\circ}$ e 9 : meses e 2 no $15^{\circ}$ mês (média $=9$ meses). Nestes pacientes, foram realizados 40 enxertos de safena (10 para descendente anterior, 3 para ramos diagonais, 13 para marginal esquerda e 14 para coronária direita) e 7 anastomoses da artéria mamária interna esquerda para a descendente anterior. O número médio de enxertos, por pacientes, foi de 2,6.

As características destes grupos estão discriminadas na Tabela 1.

\section{RESULTADOS}

Os pacientes tratados com CRM após a reperfusão coronária, independentemente da técnica de reperfusão

\section{TABELA 1}

\begin{tabular}{lcc}
\hline & Grupo I & Grupo II \\
Idade média & 56 & 55 \\
Sexo - Masculino & 24 & 14 \\
Feminino & 3 & 4 \\
IAM Prévio & 8 & 0 \\
CRM Prévia & 4 & 1 \\
Local do IAM - Anterior & 9 & 10 \\
Inferior & 18 & 8 \\
Art. Tratada - D. Anterior & 9 & 9 \\
$\quad$ C. Direita & 12 & 5 \\
$\quad$ Circunflexa & 3 & 3 \\
P. Safena & 3 & 1 \\
No. de Vasos Acometidos & & \\
Uniarterial & 1 & 3 \\
Biarterial & 3 & 4 \\
Triarterial & 23 & 11 \\
\hline
\end{tabular}

empregada, evoluíram, na fase hospitalar, sem intercorrências relacionadas ao procedimento, quais sejam, distúrbios hemorrágicos graves naqueles em que se empregou STK (inclusive nos submetidos a cirurgia nas primeiras 24 horas), ou dissecção da artéria coronária naqueles submetidos a AC.

A mortalidade, na fase hospitalar, foi de $4 / 45$ (9\%), sendo que ocorreram $2(7,4 \%)$ óbitos no Grupo I ( 1 por choque cardiogênico e 1 por arritmia ventricular grave, refratária ao tratamento intensivo) e $2(11 \%)$ óbitos no Grupo II (1 no transoperatório, por insuficiência miocárdica e 1 por hemorragia digestiva alta no $5^{\circ}$. dia de pós-operatório).

Nāo foram diagnosticados no pós-operatório imediato e, mesmo, após a alta hospitalar, reoclusōes das artérias recanalizadas ou reinfartos.

Entre os 41 (91\%) pacientes que obtiveram alta hospitalar, encontram-se assintomáticos $21(84 \%)$ no Grupo I, $15(94 \%)$ no Grupo II, com angina de graus I e II $3(8 \%)$ no Grupo I e 1 ( $2 \%)$ no Grupo II, ocorrendo $1(2 \%)$ óbito em paciente pertencente ao Grupo I, com 14 meses de evolução, por morte súbita.

Foram reestudados angiograficamente $8(32 \%)$ integrantes do Grupo I, em um período médio de 30 dias após a CRM. Todos os 19 enxertos de safena e 1 anastomose de artéria mamária interna esquerda realizados nestes doentes encontravam-se permeáveis. Houve meIhora na fração de ejeção global do ventrículo esquerdo, como demonstra a Tabela 2.

\section{DISCUSSÃO}

A reperfusão coronária é um método que vem, progressivamente, sendo empregado no tratamento do IAM. 
MATTOS, L. A.; PIEGAS, L. S.; PINTO, I. F.; TANAJURA, L. F. L.: MORAES. A. G.: PIMENTEL. W.: BUCHLER. J.: DINKHUYSEN. J. J.: SOUZA, L. C. B.; PAULISTA, P. P.; JATENE, A. D.: SOUSA, J. E. M. R. - Revascularização cirúrgica após reperfusão no infarto agudo do miocárdio. Rev. Bras. Cir. Cardiovasc. 20(2):98-101, 1987.

\section{TABELA 2}

\begin{tabular}{ll}
\hline Grupo II & $\# 8 / 25$ (32\%) \\
Fração de Ejeção Global do VE & Pré STK \\
Pós STK & $-40+3 \%$ \\
Pós CRM & $-45+9 \%$ \\
\hline
\end{tabular}

Quando se observam alguns critérios na indicação, aumenta o número de resultados positivos, beneficiando a preservação da função ventricular. Os principais critérios relatados ${ }^{2,3,10}$ são a precocidade da intervenção na fase aguda, quer com o uso da STK ou da AC, e o tratamento da lesão residual, extremamente freqüente e severa após a desobstrução coronária. SUTTON et alii ${ }^{11}$ relatam incidência mais elevada de reinfartos e reoclusōes nos pacientes que não completaram a revascularização, permanecendo com lesão residual severa. O'NEIL et alii ${ }^{7}$ e PIEGAS et alii ${ }^{8}$ demonstram, em seus trabalhos, que apenas a recanalização com sucesso não é suficiente para promover real melhora na função ventricular, pois a lesão estenótica grave persiste, sendo necessário complementar o tratamento com o emprego da CRM, ou AC.

A cirurgia tem sido empregada no tratamento da lesão residual e/ou tratamento de outras lesōes arteriais, não relacionadas à área de infarto. Preferencialmente, é reservada aos pacientes multiarteriais ${ }^{6}$.

Como é previsível, existe um maior número de pacientes operados no Grupo I, pois a STK, por si só, não é capaz de eliminar a lesão residual. No Grupo II, a CRM foi necessária apenas quando a $A C$ nāo teve sucesso, nos casos com recanalização insatisfatória, naqueles com reestenose durante a evolução, ou na presença de lesões severas em outros segmentos arteriais.

O intervalo entre a reperfusão e a cirurgia, assim como as características clínicas e angiográficas, com excessão da presença de infarto prévio, nāo diferiram, nestes dois grupos (Tabela 1).

A cirurgia precoce, nas primeiras 24 horas, foi realizada nas situaçōes de instabilidade clńica e hemodinâmica, ou na presença de lesōes sub-oclusivas. Quan- do possivel, optou-se por realizar a CRM num intervalo médio de 7 a 10 dias. A indicação de cirurgia tardia baseou-se na evoluçāo clínica e nos achados angiográficos destes doentes.

A mortalidade hospitalar, como já relatado, foi de $2 / 24(7,4 \%)$ e $2 / 12(11 \%)$ no Grupo I e Grupo II, respectivamente. Se considerarmos, independentemente dos grupos a que pertencem, que estes são pacientes infartados de elevado risco (multiarteriais, com função ventricular deprimida, portadores de infarto prévio, ou revascularizações cirúrgicas anteriores), podemos aceitar, como bastante satisfatórias, as mortalidades encontradas, ainda inferiores às obtidas com o tratamento convencional do IAM, não diferindo dos resultados publicados por outros grupos ${ }^{11},{ }^{12}$.

A evolução clínica, após a alta hospitalar, foi boa. Verifica-se que a grande maioria está assintomática, 21 (84\%) no Grupo I e 15 (94\%) no Grupo II, num seguimento médio de 27 meses. Ocorreu apenas um óbito tardio (2,4\%), no Grupo I, no 14 : mês de evolução. Em ambos os grupos, nāo foram diagnosticados reinfarto, ou reoclusāo, o que pode ser atribuido ao tratamento pleno da doença.

Foram reestudados precocemente, através da cinecoronariografia, 8 pacientes. Estavam assintomáticos e foram reestudados, no início da experiência, visando comprovar a eficácia da técnica. Nāo se observou nenhum caso de oclusāo dos enxertos ou da anastomose. Acreditamos que a evolução tardia destes doentes será semelhante àquela observada nas CRM eletivas, visto que, na fase inicial, não ocorreu nenhuma oclusão.

O comportamento da fraçāo de ejeção mostrou-se favorável, sendo, sempre, maior após a $\mathrm{CRM}^{2}, 5,7,8$. (Tabela 2).

Concluímos que a cirurgia de revascularização do miocárdio é capaz de complementar os resultados obtidos com a reperfusão coronária precoce, independentemente da técnica empregada. Permite a estes doentes uma boa evolução clínica e expressiva diminuiçăo na incidência de reinfartos e reoclusōes imediatas ${ }^{11}$. A mortalidade ainda um pouco elevada é aceitável, devido à gravidade dos pacientes tratados. Finalmente, a técnica de reperfusão miocárdica empregada não influiu nos resultados encontrados após a CRM. 
MATTOS, L. A.: PIEGAS. L. S.: PINTO, I. F.: TANAJURA, L. F. L.: MORAES. A. G.: PIMENTEL. W.: BUCHLER, J : DINKHUYSEN. J. J.: SOUZA, L. C. B.: PAULISTA, P. P.: JATENE, A. D.: SOUSA. J. E. M. R. - Revascularização cirúrgica após reperfusão no infarto agudo do miocárdio. Rev. Bras. Cir. Cardiovasc. 20(2):98-101. 1987

RBCCV $44205-28$

MATTOS, L. A.: PIEGAS, L. S.; PINTO, I. F.: TANAJURA, L. F. L.: MORAES, A. G.: PIMENTEL, W.: BUCHLER, J.; DINKHUYSEN, J. J.; SOUZA, L. C. B.: PAULISTA, P. P.; JATENE A. D.: SOUSA. J. E. M. R. - Surgical revascularization following SK or PTCA reperfusion in acute myocardial infartion. Rev. Bras. Cir. Cardiovasc., 2(2): 98-101, 1987

ABSTRACT: The coronary artery bypass surgery is frequently used to treat the residual lesion after myocardial infarction reperfusion. Patients may undergo surgery during the acute or late phase. This study analyses the results of $45 / 159(28 \%)$ patients who underwent a coronary bypass surgery after successful myocardial reperfusion with streptokinase or percutaneous transluminal coronary angioplasty (PTCA). Patients were divided into two groups accordingly to the reperfusion technique: I) $27(60 \%)$ reperfused with the use of streptokinase, intracoronary in $22(81 \%)$ and intravenous in $5(18 \%)$; II) $18(40 \%)$ reperfused with the aid of PTCA, alone in $10(55 \%)$ and following previous streptokinase in $8(45 \%)$. Patientes were analysed at the moment of surgery, clinical evolution, late cinecoronariography and death, and were followed from 2 to 55 months. Authors analise the results and the capacity of bypass surgery complement sưccessfull myocardial reperfusion during acute myocardial infarction.

DESCRIPTORS: myocardial infarct, surgery; myocardial revascularization, surgery.

\section{REFERÊNCIAS BIBLIOGRÁFICAS}

1 BERG, R.; SELINGER, S. L.; LEONARD, J. L.; GRUNWALD, R. P.; O'GRADY, W. P. - Immediate coronary artery bypass for acute evolving myocardial infarction. J. Thorac. Cardiovasc. Surg., 81 (4): 493, 1981.

2 BLANKE, H.; SCHICHA, H.; COHEN, M.; KAISER, H.; KARSCH, K. R.; NEUMANN, P.; RENTROP, K. P. Long-term follow-up after intracoronary streptokinase therapy for acute myocardial infarction. Am. Heart J., 110 (4): 736, 742, 1985.

3 BRAUNWALD, W. - The aggressive treatment of acute myocardial infarction. Circulation, 71 (6): 1087-1092, 1985.

4 HARTZLER, G. O.: RUTHERFORD, B. D.: MCCONAHAY, D. R.; JONHSON, W. L.; MC CALLISTER, B. D.; GURA, G. M. CONN, R. C.; CROCKETT, J. E. - Percutaneous transluminal coronary angioplasty with and without trombolytic therapy for treatment of acute myocardial infarction. Am. Heart J., 106 (5): 965-973, 1983.

5 KENNEDY, J. W.; RITCHIE, J. L.; DAVIS, K. B.; STADIUS, M. L.; MAYNARD, C.; FRITZ, J. - The Western Washington randomized trial of intracoronary streptokinase in acute myocardial infarction. A 12-month follow-up report. N. Eng. J. Med., 312 (17): 1073-1078, 1985.

6 MATTOS, L. A. P.; PIEGAS, L. S.; TANAJURA, L. F.; PINTO, I. M. F.; MORAES, A. G.; BEMBOM, J. C.; BUCHLER, J.; PIMENTEL, W. A,; SOUZA, L. C. B.; PAULISTA, P. P.; JATENE, A. D.; SOUSA, J. E. M. R. - Revascularizaçāo cirúrgica após estreptoquinase no infarto agudo do miocárdio. Arq. Bras. Cardiol., 47 (Supl. 1): 97, 1986. (Resumo)
7 O'NEIL, W.; TIMMIS, G. C.; BOURDILLOW, P. D.; LAI, P.: PITT, B. - A prospective randomized clinical trial of intracoronary streptokinase versus coronary angioplasty for acute myocardial infarction. N. Eng. J. Med., 314 (13): 812-818, 1986.

8 PIEGAS, L. S.; PINTO, I. M. F.; MATTOS, L. A. P.; TANAJURA, L. F.; MORAES, A. G.; TIMERMAN, A.; BUCHLER, J. R.; PIMENTEL, W. A.; SOUZA, L. C. B.; PAULISTA, P. P.; JATENE, A. D.; SOUSA, J. E. M. R. - Evolução tardia de pacientes reperfundidos com estreptoquinase intracoronária. Arq. Bras. Cardiol., 45 (Supl. 1): 63, 1985. (Resumo)

9 PRIDA, X. E.; HOLLAND, J. P.; FELDMAN, R. L.; HILL, J. A.; MACDONALD, R. G.; CONTI, R.; PEPINE, C. J. - Percutaneous transluminal coronary angioplasty in evolving acute myocardial infarction. Am. J. Cardiol., 57 (13): 1069-1074, 1986.

10 SPANN, J. F.; SHERRY, S.; CARABELLO, B. A.; DENENBERG, B. S.; MANN, R. H.; MCCANN, W. D.; GAULT, J. H.; GENTZLER, R. D.; BELBEE, A. D.; MAURER, A. H.; COOPER, E. M. - Coronary thrombolysis by intravenous streptokinase in acute myocardial infarc. tion: acute and follow-up studies. Am. J. Cardiol., 53 (6): 655-661, 1984

11 SUTTON, J. M.; TAYLOR, G. J.; MIKELL, F. L.; MOSES, H. W.; DOVE, J. T.; BATCHELDER, J. E.; WELLONS, H. A.; SCHNEIDER, J. A. - Thrombolytic therapy followed by early revascularization for acute myocardial infarction. Am. J. Cardiol., 57 (15): 1227-1231, 1986.

12 WALKER, W. E.; SMALLING, R. W.; FUENTES, F.; GOULD, K. L.; JOHNSON, W. E.; REDUTO, L. A.; STERLING, R. P.; WEILAND, A. P.; WYNN, M. M. - Role of coronary artery bypass surgery after intracoronary streptokinase infusion for myocardial infarction Am. Heart J., 107 (4): 826-829, 1984. 